\title{
Flow tracing as a tool set for the analysis of networked large-scale renewable electricity systems
}

\author{
Jonas Hörscha,, Mirko Schäfer ${ }^{\mathrm{b}, \mathrm{a}}$, Sarah Becker ${ }^{\mathrm{c}, \mathrm{d}}$, Stefan Schrammª, \\ Martin Greiner ${ }^{b}$ \\ ${ }^{a}$ Frankfurt Institute for Advanced Studies, 60438 Frankfurt am Main, Germany \\ ${ }^{b}$ Department of Engineering, Aarhus University, 8000 Aarhus C, Denmark \\ ${ }^{c}$ Department of Electrical Engineering and Computer Science, Kassel University, \\ $34125 \mathrm{Kassel}$ \\ ${ }^{d}$ Fraunhofer IWES, 34119 Kassel, Germany
}

\begin{abstract}
The method of flow tracing follows the power flow from net-generating sources through the network to the net-consuming sinks, which allows to assign the usage of the underlying transmission infrastructure to the system participants. This article presents a reformulation that is applicable to arbitrary compositions of inflow appearing naturally in models of large-scale electricity systems with a high share of renewable power generation. We propose an application which allows to associate power flows on the grid to specific regions or generation technologies, and emphasizes the capability of this technique to disentangle the spatio-temporal patterns of physical imports and exports occurring in such systems. The analytical potential of this method is showcased for a scenario based on the IEEE 118 bus network.
\end{abstract}

Keywords: System analysis and design, renewable power generation, power transmission, line cost allocation, flow tracing

\section{Nomenclature}

Indices and Labels

$n, m, k$ Index of buses.

$l, l^{\prime} \quad$ Index of lines.

$\alpha, \beta, \tau$ Labels of regions and technologies for grouping the power injection and flows.

Constants, Variables and Functions

$P_{n}(t) \quad$ Net power injection at bus $n(\mathrm{MW})$.

\footnotetext{
${ }^{*}$ Corresponding author

Email address: hoersch@fias.uni-frankfurt.de (Jonas Hörsch)
} 


\begin{tabular}{|c|c|}
\hline$G_{n}^{\tau}(t)$ & Power generation by technology $\tau$ at bus $n$ (MW). \\
\hline$L_{n}(t)$ & Load at bus $n(\mathrm{MW})$. \\
\hline$F_{n \rightarrow m}^{\text {out }}(t)$ & Power outflow from bus $n$ in direction of bus $m(\mathrm{MW})$. \\
\hline$F_{n \rightarrow m}^{i n}(t)$ & Power inflow to bus $m$ from bus $n$ (MW). \\
\hline$F_{l}(t)$ & Absolute value of the power flow on line $l$. \\
\hline$\chi_{n \rightarrow m}(t)$ & Loss in the transmission line between bus $n$ and $m$ (MW). \\
\hline$q_{n, \alpha}^{i n}(t)$ & $\begin{array}{l}\text { In-partition, the share of the injected power at bus } n \text { attributed } \\
\text { to owner } \alpha \text {. }\end{array}$ \\
\hline$q_{n, \alpha}^{\text {out }}(t)$ & $\begin{array}{l}\text { Out-partition, the share of the consumed power at bus } n \text { at- } \\
\text { tributed to owner } \alpha \text {. }\end{array}$ \\
\hline$q_{l, \alpha}(t)$ & $\begin{array}{l}\text { Line-flow partition, the share of the power flow through line } l \\
\text { attributed to owner } \alpha \text {. }\end{array}$ \\
\hline$p_{l}\left(F_{l}\right)$ & Probability for a flow $F_{l}$ on line $l$. \\
\hline$p_{l}\left(q_{l, \alpha} \mid F_{l}\right)$ & $\begin{array}{l}\text { Conditional probability for a share } q_{l, \alpha} \text { of owner } \alpha \text { in case of a } \\
\text { flow } F_{l} \text {. }\end{array}$ \\
\hline$h_{l, \alpha}\left(F_{l}\right)$ & Average share of owner $\alpha$ on the link $l$ for a flow $F_{l}$. \\
\hline$w_{l, \alpha}(\mathcal{K})$ & $\begin{array}{l}\text { Weight for the usage of the capacity increment between } \mathcal{K} \text { and } \\
d \mathcal{K} \text { attributed to owner } \alpha \text { on the link } l .\end{array}$ \\
\hline $\mathcal{K}^{T}$ & Transmission capacity of the network (MW). \\
\hline $\mathcal{K}_{l}^{T}$ & Transmission capacity of line $l(\mathrm{MW})$. \\
\hline$\overline{\mathcal{K}}^{T}$ & $\begin{array}{l}\text { Transmission capacity of the network including length (MW } \\
\mathrm{km} \text { ). }\end{array}$ \\
\hline$\overline{L_{l}}$ & Length of transmission line $l(\mathrm{~km})$. \\
\hline $\bar{D}_{n}$ & Average graph distance of bus $n(\mathrm{~km})$. \\
\hline $\mathcal{M}_{\alpha, \tau}^{(1 \ldots 4)}$ & Transmission network usage measures (MW km). \\
\hline
\end{tabular}

\section{Introduction}

The electricity system is built up of a complex interwoven network of technologies, which provides the backbone for our modern society. In the past, this network was characterized by power flows from large central power plants downstream through the grid to the consumers, with only very limited interactions between different geographical regions. Today, the rising share of decentralized, fluctuating renewable generation and the increasing inter-dependence of international electricity markets has led to a more dynamical system: the power grid has become the underlying infrastructure for a complex pattern of longrange power flows between a heterogeneous distribution of power generation to consumers, integrating not only dispatchable conventional generation, but also electricity from offshore wind farms, wind and solar parks and roof-top solar panels. In this context, a deeper understanding of the emerging power flow patterns is of paramount importance on different levels: For instance, internationally integrated electricity markets need to incorporate possible network congestion into their market design [1], whereas network expansion plans attempt to minimize this congestion in the long run $[2,3]$. Also the delevopment of fair and transparent grid usage fee systems, or public discussions concerning 
the benefit of new infrastructure projects rely strongly on insights concerning the composition and dynamics of the flow pattern in the network $[4,5]$. In this article we present a reformulation of a well-known method of flow allocation, denoted as average participation or flow tracing, that is well adapted to the challenges of the system analysis of complex modern electricity systems. Different approaches to the problem of flow allocation in power grids are often derived from circuit theory $[6,7]$ or are based on approximations of the complex power flow equations for AC electrical networks [8,9]. For the application of such methods to the problem of flow allocation in large-scale models of electricity systems, one has to factor in the potentially coarse-grained nature of such models. Both the network buses and transmission lines might be aggregated representations of lower level infrastructures, which cannot be included in detail in the model due to computational limitations or lack of data [10-12]. The method of flow tracing can be applied directly to the overall power flow pattern in the system, and thus does not explicitly have to take into account the underlying modeling details. By tracing what we term in-partitions, we show how the known composition of network-injected power generation can be followed through the grid and thus be transferred to the power flows and composition of net consumption at the sink nodes. In this way the location of generation of power flow can be connected to its location of consumption, thus disentangling the complex spatio-temporal patterns of imports and exports inherent to interconnected electricity systems with a high share of renewable generation. We showcase the potential of this methodological tool set by application to the Scenario 2023B of the IEEE 118-bus model adapted by Barrios et al. at RWTH Aachen with renewable generation capacities and hourly availability for a model year as a benchmark for transmission expansion algorithms [13].

After a short review of flow tracing, Sec. 2 introduces the reformulated flow tracing technique and a measure of network usage. The subsequent Sec. 3 showcases two exemplary applications: Firstly the tracing of power flow of different generation types between several regions across a network model based on the IEEE 118 bus case, and secondly a comparison of a statistical transmission capacity usage measure with several alternative allocation mechanisms. Section 4 concludes the paper.

\section{Methodology}

Flow tracing was introduced as a loss-allocation scheme by Bialek et al. based on solving linear equations [14] and in parallel by Kirschen et al. as an analytical tool using a graph-based, iterative approach [15].

It was soon after proposed as a transmission-usage allocation scheme [16-19]. Subsequently, the method was discussed to cover concrete supplementary charge schemes for cross-border trades [20,21], in view of the discussion about the mechanism of inter-transmission system operator compensation in Europe [4, $22,23]$.

Of the other network-cost allocation methods - reviewed in [24] or [25], for instance - we only want to highlight marginal participation [26], which attributes 


\begin{tabular}{rlrrrrrrr}
\hline$n$ & $G_{n}$ & \multicolumn{2}{c}{ of which in \% } & & $L_{n}$ & $G_{n}-L_{n}$ & $I_{n}$ & $X_{n}$ \\
& & wind & solar & other & & & & \\
\hline 1 & 76.0 & 16 & 19 & 65 & 65.5 & 10.5 & 0.9 & 5.6 \\
2 & 20.5 & 8 & 0 & 92 & 21.1 & -0.6 & 0.9 & 0.6 \\
3 & 8.5 & 2 & 13 & 85 & 8.0 & 0.5 & 0.0 & 1.8 \\
4 & 7.3 & 12 & 6 & 82 & 7.5 & -0.3 & 0.0 & 2.5 \\
\hline
\end{tabular}

Table 1: Power generation and consumption of a simple four bus network with im-/exports with external buses in GW.

transmission capacity according to linear sensitivities of network flows to differential bus injections as captured by the power transfer distribution factors (PTDF) [27]. Due to its influence on the PTDF, for this method the choice of the slack bus has to be taken into account explicitly [28], whereas for the flow tracing technique this choice only affects the total power flow but not the allocation mechanism.

\subsection{Power flow}

The active power flow in an electricity system satisfies Kirchhoff's current law. If the net power injection at bus $n$ from generators and loads is given by $P_{n}$, and $F_{n \rightarrow m}^{i n / o u t}$ are the power in- and outflows from bus $n$ to $m$, then the power flow through node $n$ is conserved as

$$
P_{n}^{i n}+\sum_{m} F_{m \rightarrow n}^{i n}=P_{n}^{o u t}+\sum_{m} F_{n \rightarrow m}^{o u t} .
$$

Here we use the positive and negative injections $P_{n}^{\text {in }}$ and $P_{n}^{\text {out }}$ at node $n$ and invoke the convention that all $F_{m \rightarrow n}^{o u t}$ and $F_{m \rightarrow n}^{i n}$ are positive or zero.

Table 1 introduces a particular snapshot in a simple network with four buses with generation $G_{n}$, load $L_{n}$ and im-/exports $I_{n} / X_{n}$ with other buses not represented explicitly. In this example, we take the positive injection as the net surplus between generation $G_{n}$ and demand $L_{n}$ plus the imports $I_{n}$, while the negative injection follows from the deficit and exports $X_{n}$, as

$$
P_{n}^{\text {in }}=\max \left\{\left(G_{n}-L_{n}\right), 0\right\}+I_{n}, \quad P_{n}^{\text {out }}=\max \left\{-\left(G_{n}-L_{n}\right), 0\right\}+X_{n} .
$$

The flows and line-losses are illustrated in Fig.1. The convention means that the line from bus 1 to bus 3 is described by $F_{1 \rightarrow 3}^{o u t}=2.2 \mathrm{GW}, F_{1 \rightarrow 3}^{i n}=1.8 \mathrm{GW}$, $F_{3 \rightarrow 1}^{\text {in }}=0$ and $F_{3 \rightarrow 1}^{\text {out }}=0$.

Here and in general the outflow from bus $n$ to $m, F_{n \rightarrow m}^{\text {out }}$, is larger than the inflow to $m, F_{n \rightarrow m}^{i n}$ due to losses in the transmission line $n \rightarrow m$. We denote them by $\chi_{n \rightarrow m}=F_{n \rightarrow m}^{\text {out }}-F_{n \rightarrow m}^{\text {in }}$.

\subsection{Flow tracing}

The flow tracing method by Bialek and Kirschen $[14,15]$ follows the power flow from individual buses through the network and decomposes the flow on 
Figure 1: Power flows and injections in the simple four bus network introduced in Table 1 in units of GW. Injections are composed of generation, consumption, im- and exports as described in the text

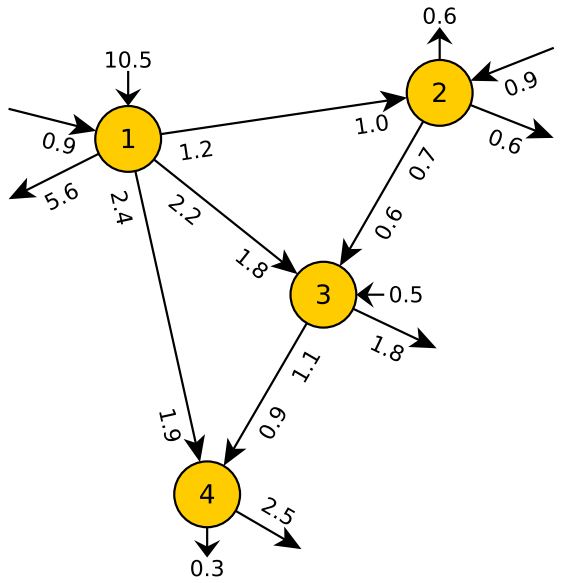

the power lines into contributions associated to each bus. Since for large-scale electricity systems, the injection $P_{n}^{i n}$, in general, already contains several constituents, we introduce an in-partition $q_{n, \alpha}^{i n}$ associating the power injection at each bus $n$ to a set of components $\alpha$. For the power flows of the four bus example, we will use the components $\{1,2,3,4, I\}$ with the in-partition

$q_{n, \alpha}^{i n}=\left\{\begin{array}{ll}\frac{\max \left\{G_{n}-L_{n}, 0\right\}}{P_{n}^{i n}} & \text { for } \alpha=n \wedge P_{n}^{i n}>0, \\ \frac{I_{n}}{P_{n}^{i n}} & \text { for } \alpha=I \wedge P_{n}^{i n}>0, \\ 0 & \text { else. }\end{array}\right\}=\left(\begin{array}{ccccc}\frac{10.5}{11.4} & 0 & 0 & 0 & \frac{0.9}{11.4} \\ 0 & 0 & 0 & 0 & 1 \\ 0 & 0 & 1 & 0 & 0 \\ 0 & 0 & 0 & 0 & 0\end{array}\right)$

to differentiate the imports $I_{n}$ entering at each bus from the power generated there. Note that the component $I$ is associated with injected power throughout the network. Similarly, another in-partition for components \{wind, solar, other, imports\} is able to encode the relative shares of wind, solar and other energy generation sources from Table 1.

Flow tracing follows the diffusion of the different components $\alpha$ by assuming conservation of the partial power flows at bus $n$ in analogy to (1)

$$
q_{n, \alpha}^{\text {in }} P_{n}^{\text {in }}+\sum_{m} q_{m, \alpha}^{(m \rightarrow n)} F_{m \rightarrow n}^{\text {in }}=q_{n, \alpha}^{(\text {out })} P_{n}^{\text {out }}+\sum_{m} q_{n, \alpha}^{(n \rightarrow m)} F_{n \rightarrow m}^{\text {out }} .
$$

In general there is a degree of freedom in relating $q_{n, \alpha}^{(o u t)}$ and $q_{n, \alpha}^{(n \rightarrow m)}$ under the boundary condition of assuring conversation of partial flows. It is nevertheless intuitive to assume that the power contributions mix perfectly in each bus and the partitions of the flows leaving a bus are all identical $q_{n, \alpha}=q_{n, \alpha}^{(\text {out })}=q_{n, \alpha}^{(n \rightarrow m)}$. This assumption equally underlies the tracing by Bialek and Kirschen and is known as proportional sharing. Bialek et. al. were able to show the proportional sharing principle to coincide with the Shapley value of a stylized game of loss attribution played by two generators feeding into the same line [29], but it can 
rightfully be contested for practical purposes. For the purpose of flow allocation in large-scale electricity models, we suggest this realization in particular due to its intuitiveness and lack of additional parameters. With the proportional sharing assumption Eq. (4) reduces to a system of $N \times A$ equations for $N \times A$ unknowns $q_{n, \alpha}$

$$
q_{n, \alpha}^{i n} P_{n}^{i n}+\sum_{m} q_{m, \alpha} F_{m \rightarrow n}^{i n}=q_{n, \alpha}\left(P_{n}^{\text {out }}+\sum_{m} F_{n \rightarrow m}^{\text {out }}\right),
$$

with $A$ denoting the number of components $\alpha$. If we eliminate inert buses without any flows from the network (without any loss of generality) and abbreviate the power leaving a bus as nodal flow $F_{n}:=P_{n}^{o u t}+\sum_{k} F_{n \rightarrow k}^{o u t}$, we can rearrange Eq. (5) to

$$
q_{n, \alpha}^{i n} P_{n}^{i n}=F_{n} \sum_{m}\left[\delta_{n, m}-\frac{F_{m \rightarrow n}^{i n}}{F_{n}}\right] q_{m, \alpha}
$$

with the Kronecker delta $\delta_{n, m}$. Finally, with the definition of the matrix

$$
D_{n, m}=\frac{F_{m \rightarrow n}^{i n}}{F_{n}}
$$

capturing the share that the power from bus $m$ contributes to the nodal flow through bus $n$, Eq. (6) can be rendered in matrix notation as

$$
\operatorname{diag}\left(P^{i n}\right) q^{i n}=\operatorname{diag}(F)(1-D) q
$$

For a lossless power flow, $F_{n \rightarrow m}^{\text {out }}=F_{n \rightarrow m}^{i n}, 1-D$ is the transpose of the downstream distribution matrix $A_{d}$ in Bialek's formulation and together with $q^{i n}=q=1$ the proposed method reduces to Bialek's flow tracing. The steps from Eq. (5) to Eq. (8) illustrate the equivalence of the two formulations of flow tracing discussed in the literature as a linear algebra problem [14] and a graph-based algorithm [15].

Eq. (8) is solved formally as

$$
q=(1-D)^{-1} \operatorname{diag}\left(P^{i n} / F\right) q^{i n},
$$

where the inverse of $1-D$ can be shown to exist as Neumann series (1 $D)^{-1}=\sum_{k=0}^{\infty} D^{k}$, since the absolute value of each eigenvalue of $D$ is smaller than 1, if there is at least one bus with a positive power injection in each connected component, similarly to [30]. While, therefore, the method is formally applicable also in the presence of loop flows, the interpretation of the resulting flow attribution still remains to be investigated.

To apply flow tracing to the 4-bus example shown in Fig. 1 one calculates the in-partition $q^{i n}$ (Eq. (3)), nodal flows $F$, power injections $P^{i n}$ and matrix 
$1-D$

$$
\begin{aligned}
F & =\left(\begin{array}{llll}
11.4 & 1.9 & 2.9 & 2.8
\end{array}\right), \\
P^{i n} & =\left(\begin{array}{llll}
11.4 & 0.9 & 0.5 & 0
\end{array}\right), \\
1-D & =\left(\begin{array}{cccc}
1 & 0 & 0 & 0 \\
-1 / 1.9 & 1 & 0 & 0 \\
-1.8 / 2.9 & -0.6 / 2.9 & 1 & 0 \\
-1.9 / 2.8 & 0 & -0.9 / 2.8 & 1
\end{array}\right) .
\end{aligned}
$$

By evaluation of Eq. (9), one then finds

$$
q \approx\left(\begin{array}{ccccc}
0.921 & 0.0 & 0.000 & 0.0 & 0.079 \\
0.485 & 0.0 & 0.000 & 0.0 & 0.515 \\
0.672 & 0.0 & 0.172 & 0.0 & 0.156 \\
0.841 & 0.0 & 0.055 & 0.0 & 0.104
\end{array}\right)
$$

where each column corresponds to the share of each nodal flow associated with a component, and consequently also to the share on the out-going lines. Since buses 2 and 4 do not feed any power into the network, they do not contribute to any flows. The shares of bus 1 , which feeds all buses directly, are strongest at bus 1 and 4, while at bus 2 a strong in-flow by imports dilutes the share of bus 1 . Power entering the network as imports is present at every bus, making up $15.6 \%$ of the nodal flow through bus 3 and $10.4 \%$ of the nodal flow at bus 4 . The generation of bus 3 only appears at buses 3 and 4 .

Since the power loss happens on the links it is natural to attribute a loss $q_{n, \alpha} \chi_{n \rightarrow m}$ to entity $\alpha$, f.ex. according to flow tracing imported power leads to a loss of $q_{2, I}\left(F_{2 \rightarrow 3}^{\text {out }}-F_{2 \rightarrow 3}^{\text {in }}\right)=0.515 \cdot 0.1 \mathrm{GW}$ in line $2 \rightarrow 3$. Substituting $F_{m \rightarrow n}^{o u t}=F_{m \rightarrow n}^{i n}+\chi_{m \rightarrow n}$ in Eq. (5)

$$
q_{n, \alpha}^{i n} P_{n}^{+}+\sum_{m} q_{m, \alpha} F_{m \rightarrow n}^{i n}=q_{n, \alpha}\left(P_{n}^{-}+\sum_{m} \chi_{n \rightarrow m}\right)+q_{n, \alpha} \sum_{m} F_{n \rightarrow m}^{i n},
$$

reveals by comparing the structure again to Eq. (5) that this loss allocation scheme is equivalent to treating a loss on line $n \rightarrow m$ as an additional load at the out-flowing bus $n$ combined with flow tracing on the inflows indiscriminately, the procedure Bialek introduced as net flows [14].

In summary, for a given flow pattern $F_{n \rightarrow m}$ and a fixed attribution of the generated power $q_{n, \alpha}^{i n} P_{n}^{+}$to a set of components $\alpha$ the flow tracing algorithm yields the attribution of all flows along the links $q_{n, \alpha} F_{n \rightarrow m}$ and the attribution of the power flowing into the consuming nodes $q_{n, \alpha} P_{n}^{-}$.

Note that we are able to invert the injection pattern and flow graph consistently by switching the signs $P_{n}^{ \pm} \rightarrow P_{n}^{\mp}$ and the flows $F_{n \rightarrow m}^{\text {in/out }} \rightarrow F_{m \rightarrow n}^{\text {out } / \text { in }}$. This procedure allows a given out-partition to be considered as the input for the flow tracing algorithm, which then assigns shares of the power flow and injected power at the source node according to this partition. 


\subsection{A measure of transmission line usage}

The flow tracing method as displayed in the last section refers to the application to a single flow pattern. However, for the analysis of complex modern electricity systems, one rather has to consider whole time series of fluctuating injection and flow patterns taking place on the underlying power grid. The application of the flow tracing method then yields a time series $\left(F_{l}(t),\left\{q_{l, \alpha}(t)\right\}\right)$, containing the power flows $F_{l}(t)$ and the respective shares $q_{l, \alpha}(t)$ assigned to the components $\alpha$ for each link $l$. In order to derive the respective grid usage over the whole time series, this information has to be integrated into a suitable transmission capacity usage measure. Fig. 5 below illustrates the need for such a non-trivial measure in a realistic example: Consider, for instance, in cyan the shares associated with onshore wind on a specific line; while the shares shown as small dots vary strongly over time, their conditional averages,

$$
h_{l, \alpha}(F)=\left\langle q_{l, \alpha}(t)\right\rangle_{\left\{t \mid F_{l}(t)=F\right\}},
$$

depend smoothly on the absolute line flow at which the average is taken. The right plot in Fig. 5 shows a line where on average all the power transmitted at a low line-loading is traced back to conventional generators, while in hours with a high line-loading on- and offshore wind contribute nearly all the power. Such a striking difference should be accounted for as relevant information by an adequate usage measure. In the following we briefly review such a capacity usage measure introduced in [31].

Their central idea is that the transmission line capacity of a small increment between $\mathcal{K}$ and $\mathcal{K}+d \mathcal{K}$ is only used by flows $F(t)>\mathcal{K}$ and, thus, the usage share of a component for this capacity increment is determined only from those as

$$
w_{l, \alpha}(\mathcal{K})=\left\langle q_{l, \alpha}(t)\right\rangle_{\left\{t \mid F_{l}(t)>\mathcal{K}\right\}} .
$$

Mind ">" in the subscript. The capacity of the whole line $\mathcal{K}_{l, \alpha}^{T}$ can then be split for the individual components $\alpha$ by summing all increments to

$$
\mathcal{K}_{l, \alpha}^{T}=\frac{\mathcal{K}_{l}^{T}}{\max _{t} F_{l}(t)} \int_{0}^{\max _{t} F_{l}(t)}\left\langle q_{l, \alpha}(t)\right\rangle_{\left\{t \mid F_{l}(t)>\mathcal{K}\right\}} \mathrm{d} \mathcal{K} .
$$

The proportional factor in front of the integral accounts for the fact that $w_{l, \alpha}(\mathcal{K})$ vanishes at the maximum flow by sharing the remaining security margin $\mathcal{K}_{l, \alpha}^{T}-$ $\max _{t} F_{l}(t)$ proportionally, since it is important to all users of the capacity in an analogous way to the actually used capacity. Nevertheless, depending on the details of the system under investigation other schemes are possible.

\section{Flow tracing applied to a 118-bus electricity network model}

For demonstrating the application of the reformulated flow tracing methodology, we briefly introduce an electricity system model that has been developed as a benchmark for transmission expansion methods. The IEEE 118 bus network model has been geographically embedded and augmented by attaching 


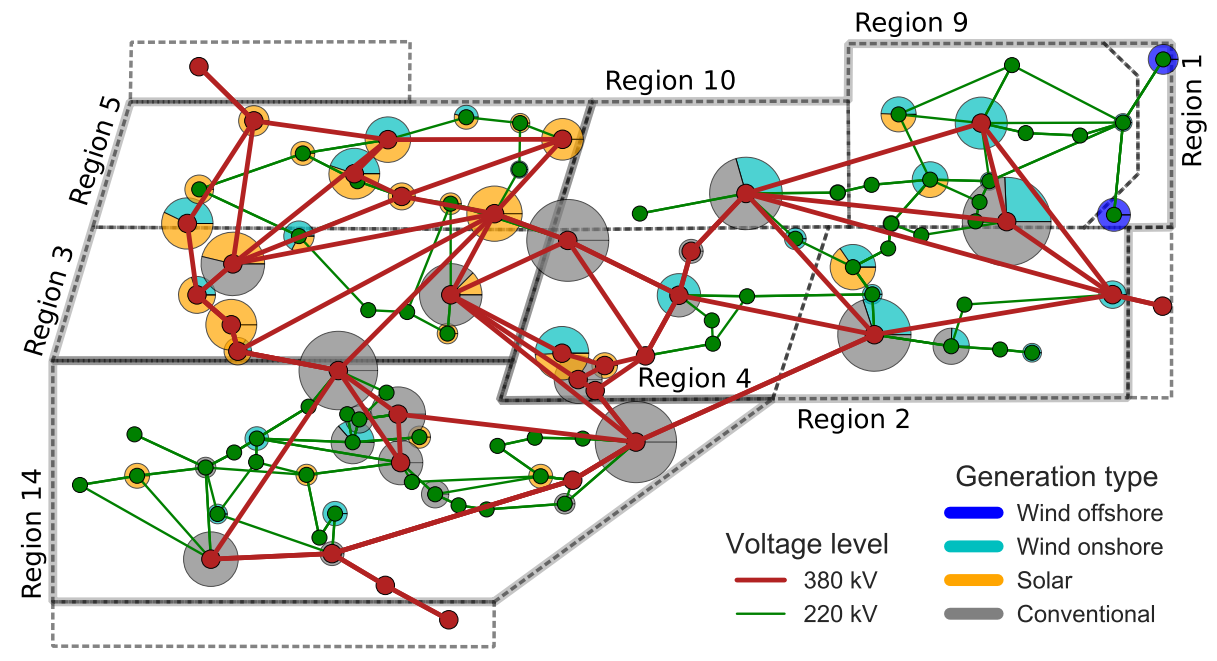

Figure 2: Scenario 2023B of the 118-bus transmission expansion benchmark case with renewable generation capacities from [13]. In the background the relative composition of the generation capacities of each region are indicated.

specific loads and conventional as well as renewable generators to the system by Barrios et al. at RWTH Aachen [13]. The load curves and the renewable generation availability span all hours in a model year. The geographic regions have weather characteristics in line with the artificial TRY-Regions of the socalled TRY reference data set of the German weather service (DWD), which feature a higher solar capacity factor in the North-West and a higher wind capacity factor in the East, where, in addition, an offshore-wind region is located. The network topology and generation capacities are shown in Fig. 2, while the average generation and consumption of each region are included in Fig. 3.

We use our electricity system modeling framework PyPSA [32] to determine the linear optimal power flow (LOPF), i.e. the dispatch of the generators is solved by a convex linear optimization minimizing the total cost based on the marginal costs of the conventional generators and the spatially and temporally fluctuating availability of renewable generation subject to meeting the load curve and the transmission constraints in all hours. Once the generator dispatch has been determined, the non-linear power flow is found by a standard NewtonRaphson iteration. Several key figures of the optimization are summarized in Table 2 .

\subsection{Analyzing mean flow patterns}

To adopt the flow tracing method one initially distinguishes the injections per region by choosing an in-partition

$$
q_{\alpha, n}^{i n}=\delta_{\alpha, n}:=\left\{\begin{array}{ll}
1 & \text { for node } n \text { in region } \alpha, \\
0 & \text { else }
\end{array} .\right.
$$




\begin{tabular}{rcccccc}
\hline & \multicolumn{3}{c}{ Generation } & & Load & Loss \\
& wind off. & wind on. & solar & conv. & & \\
\hline capacities & 1.8 & 21.2 & 22.3 & 27.9 & - & - \\
mean & 0.8 & 4.5 & 2.6 & 10.9 & 18.6 & 0.2 \\
std & 0.7 & 4.3 & 4.1 & 5.9 & 3.2 & 0.16 \\
min & 0 & 0 & 0 & 0.05 & 9.4 & 0.03 \\
$\max$ & 1.8 & 21.0 & 19.7 & 25.9 & 26.3 & 0.9 \\
\hline
\end{tabular}

Table 2: Characteristic figures of the LOPF solution in units of GW

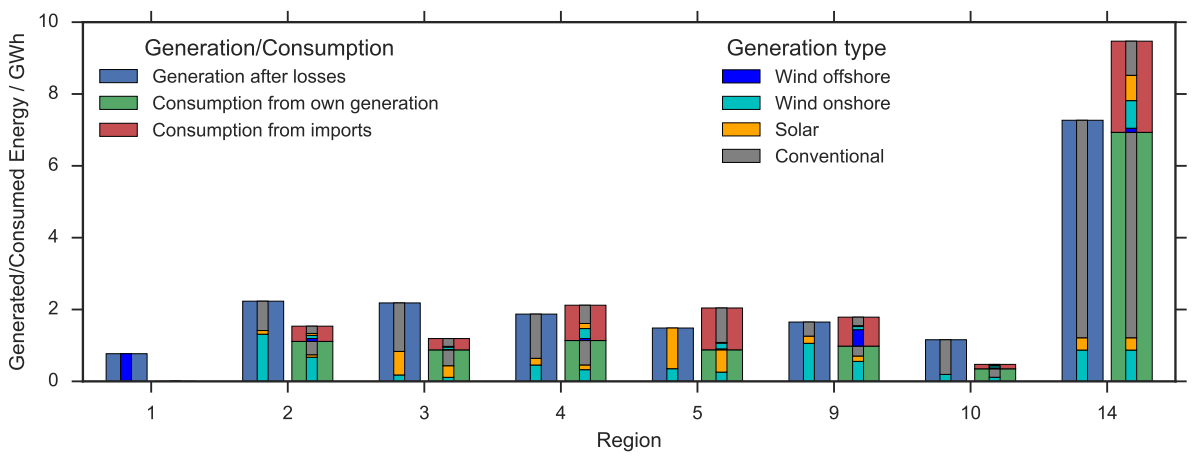

Figure 3: Comparison of the generated energy $\sum_{\beta} E_{\alpha, \beta}$ (blue) in a region with its consumption from own generation $E_{\beta, \beta}$ (green) and from imports $\sum_{\alpha \neq \beta} E_{\alpha, \beta}$ (red) dissected with flow tracing on the in-partition Eq. (18). The inset decomposition into energy per generation type has been calculated from the in-partition in Eq. (20).

Figure 4: Relative imports of a Region $\left(E_{\alpha, \beta} / \sum_{\alpha \neq \beta} E_{\alpha, \beta}\right)$. For example, the value 0.6 between Region 1 and 9 means that $60 \%$ of the consumption from imports is covered by energy generated in Region 1.

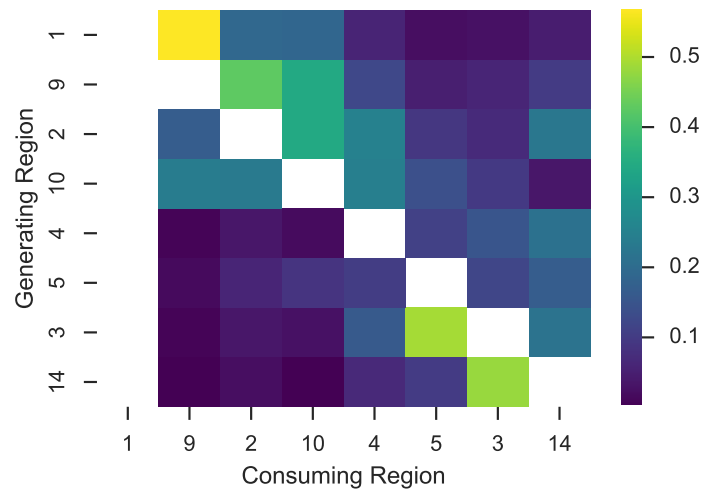


Using the flow tracing solution Eq. (9) we find the partition $q_{\alpha, n}^{\text {out }}(t)$ as the share of the energy consumed in bus $n$ that is generated in region $\alpha$, i.e. $q_{\alpha, n}(t) P_{n}^{-}(t)$. For the total amount of energy from a region $\alpha$, we only need to correct for the energy generated and consumed directly at bus $n$ given by $L_{n}-P_{n}^{-}$, if bus $n$ also belongs to region $a$. The average inter-region flow from region $\alpha$ to region $\beta$ then adds up to

$$
E_{\alpha, \beta}=\sum_{n \text { in region } \beta}\left\langle q_{\alpha, n}^{\text {out }} \cdot P_{n}^{-}+\delta_{\alpha, n} \cdot\left(L_{n}-P_{n}^{-}\right)\right\rangle_{t} .
$$

These flows are illustrated in Fig. 3 and 4.

If you ignore the inset decomposition about renewables and focus on the outer blocks in blue, red and green for now, Fig. 3 compares the net generated energy $\sum_{b} E_{\alpha, \beta}$ to the consumed energy $\sum_{\alpha} E_{\alpha, \beta}$ in each region. The consumption has been decomposed into two parts which are covered by local production and by imports. In contrast to summing up the generation independent of flow tracing as $\sum_{n}$ in region $\alpha G_{n}$, the small losses of about $5 \%$ for the energy generated in the offshore Region 1 and about $2 \%$ for the other regions have automatically been netted away by considering directly the consumed energy.

The full benefit of using flow tracing for the average flow statistics becomes only clear once we distinguish also between different generation types. We use the components $\left\{(\alpha, \tau) \mid \alpha \in\right.$ regions, $\tau \in\left\{\right.$ wind $_{\text {on }}$, wind $\mathrm{off}_{\text {off }}$, solar, other $\left.\}\right\}$ and extend the in-partition from Eq. (18) to

$$
q_{(\alpha, \tau), n}^{i n}(t)=\delta_{\alpha, n} G_{n}^{\tau}(t) / \sum_{\tau^{\prime}} G_{n}^{\tau^{\prime}},
$$

while Eq. (19) is adapted by substituting $\alpha \rightarrow(\alpha, \tau)$. The resulting measure $E_{(\alpha, \tau), \beta}$ yields the decomposition in generation types shown in Fig. 3.

Regions with only one or two types of generation capacities in the studied network model usually import a generation mix that is far more balanced. This can be observed, for instance, in Region 5, which only generates solar and wind energy, but consumes nevertheless more than a third of conventionally generated energy and Region 14 with mostly conventional generation capacities importing also a significant amount of energy from renewable generation. It is also found that the energy generated by offshore wind in Region 1 is mainly consumed (to $56 \%$ ) in the adjacent Region 9 and only a tiny amount of $5 \%$ reaches the remote Region 14.

To study the spatial pattern on imports and exports in more detail, we decompose the imports of each region further into the partial flows originating from each of the other regions in Fig. 4. The order of the regions is chosen from the North-East to the South-West highlighting two local clusters between regions 1, 9, 2 and 10 and between regions 5, 3 and 14. Region 4 has a status of its own, since it receives most of its imports from the north-eastern cluster, while it exports to the south-western cluster. The high-load Region 14 satisfies also about a fourth of its imports from regions 2 and 9 outside of its own cluster. This indicates a net flow from the North-East to the South-West not unlike the 

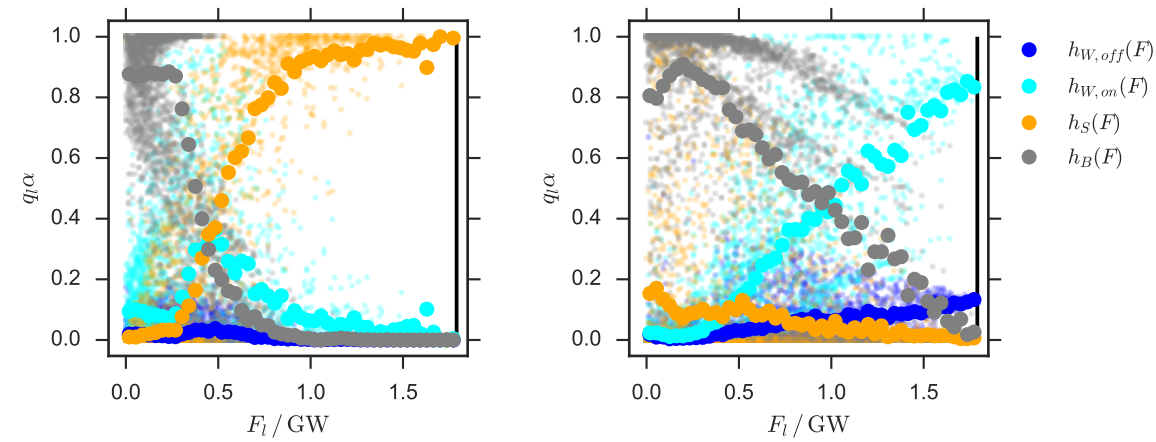

Figure 5: Usage share of two single lines: The left power line connecting Region 3 and Region 14 on the left and the power line between Region 2 and Region 14 on the right, both highlighted in Fig. 6. Data points $\left(F_{l}(t), q_{l, \alpha}(t)\right)$ for all hours and the conditional average shares $h_{l, \alpha}\left(F_{l}\right)$.

German situation of wind energy surpluses in the North-East flowing to the load-intensive South and West.

Note that while, for simplicity, we studied the average energy flows, all the partial flows are available as time-series retaining correlations to important network characteristics. The following section uses the correlations to line-loading for attributing transmission line capacity.

\subsection{Attributing transmission capacity}

In this section we will demonstrate the application of the line usage measure reviewed in Sec. 2.3 to determine the transmission capacity that is attributed to the four generation types on each link. Extending the investigations in [31], we will then compare the results to several other allocation measures. In contrast to specific cost allocation models as f.ex. Soares et al. [33] propose for pricing distribution grid capacities, our focus lies on improving the underlying usage measure, in particular by incorporating correlations to the absolute value of the power flows as detailed in Sec. 2.3.

Usage shares of the transmission lines for generation types $\tau$ are captured by the line-flow partition $\left\{q_{l, \tau}(t)\right\}$ which results from flow tracing on an in-partition

$$
q_{n, \tau}^{i n}(t)=G_{n}^{\tau}(t) / \sum_{\tau^{\prime \prime}}\left(G_{n}^{\tau^{\prime}}(t)\right),
$$

based on the hourly energy generation $\operatorname{mix} G_{n}^{\tau}(t)$.

These shares vary significantly with the flow on a power line. For both lines shown in Fig. 5 conventionally generated power has a high share only in hours with low amounts of flow. In hours with a high line-loading the line in the West is mainly occupied by energy traced back to solar panels, while the line in the East carries mostly wind power.

If one neglected this correlation for assessing the usage of the latter eastern power line, one would find that the total amount of conventional power is about 


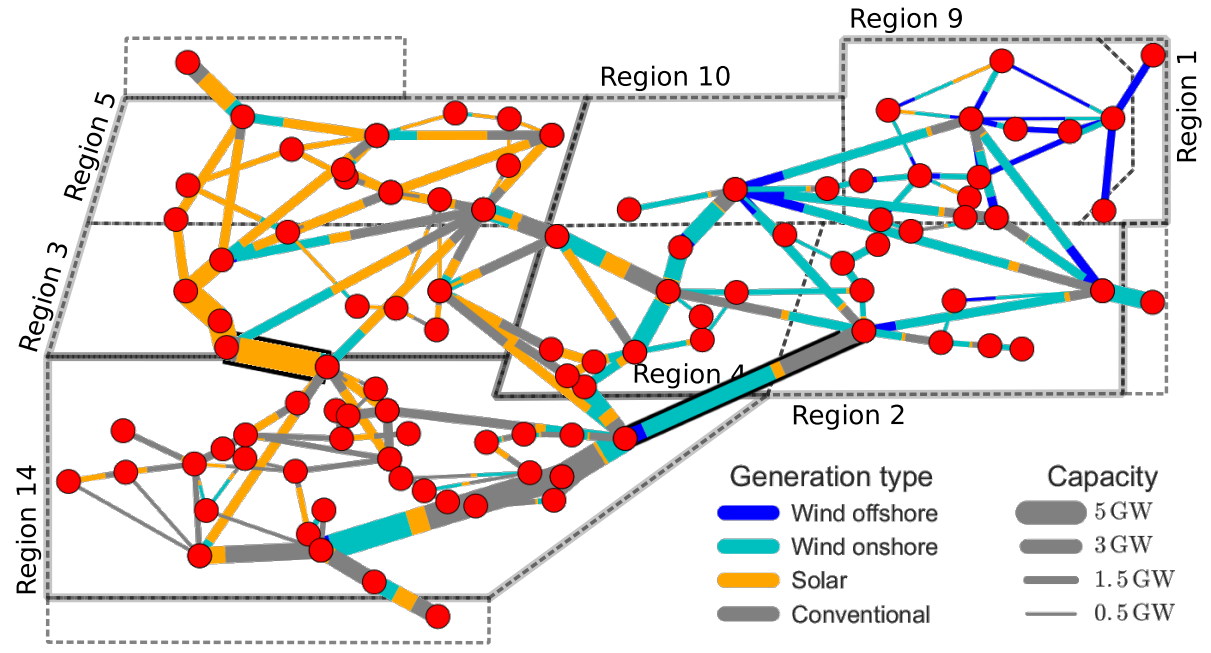

Figure 6: Line capacities $\overline{\mathcal{K}}_{l, \alpha}^{T}$ attributed to the four generation types $\alpha$ for each link $l$ throughout the network of the benchmark case. A clear east-west separation is visible: In the East the high wind generation capacity is reflected in lines primarily loaded by wind energy, while in the West solar energy is dominating. The links highlighted by a black frame have been discussed in detail in Figure 5.

a third higher than the amount of wind power flowing through it and, thus, would conclude that the costs of the power line should be split in the same proportion. Instead, the reviewed usage measure from Sec. 2.3 gives a higher weight to the shares with high line loads. To compare attributed transmission capacities in line with transmission cost drivers length and capacity, we multiply it by length $\bar{L}_{l}$ as

$$
\overline{\mathcal{K}}_{l, \alpha}^{T}=\frac{\bar{L}_{l} \mathcal{K}_{l}^{T}}{\max _{t} F_{l}(t)} \int_{0}^{\max _{t} F_{l}(t)}\left\langle q_{l, \alpha}(t)\right\rangle_{\left\{t \mid F_{l}(t)>\mathcal{K}\right\}} \mathrm{d} \mathcal{K} .
$$

Similarly we understand the total transmission capacity of the network to be given by $\overline{\mathcal{K}}^{T}=\sum_{l} \mathcal{K}_{l}^{T} \bar{L}_{l}$ in units of MW $\mathrm{km}$. The evaluation of the measure for the eastern line in Fig. 5 attributes $54 \%$ to onshore wind and only $34 \%$ to conventionally generated power.

The attributed capacities of all the transmission lines in the network are shown schematically in Fig. 6. Power from onshore wind turbines takes up most of the capacity in the East, while power generated by solar panels is attributed the transmission capacity in the West. This separation mirrors the distribution of the generation capacities (cf. Fig. 2). Renewable generation is attributed a share of the transmission capacity that is disproportionately high compared to the average energy generation mix, given in Table 2. In Region 14, for instance, where only few renewable generation capacities are located, significant amounts of transmission capacity are attributed to solar and wind generation.

We finally compare the flow tracing based usage measure in Eq. (22) with several alternative allocation mechanisms for transmission capacity: 
Average power injection splits the transmission capacity of the network $\overline{\mathcal{K}}^{T}$ in proportion to the amount of injected power of each generator $(n, \tau)$, i.e.

$$
\mathcal{M}_{\alpha, \tau}^{(1)}=\left(\frac{\sum_{n \text { in region } \alpha}\left\langle P_{n, \tau}^{+}\right\rangle_{t}}{\sum_{m}\left\langle P_{m, \tau}^{+}\right\rangle_{t}}\right) \overline{\mathcal{K}}^{T},
$$

where $P_{n, \tau}^{+}(t)$ is the power injected by a generation type $\tau$ at bus $n$. This scheme corresponds to the widely used postage stamp pricing mechanism.

Average power injection with topological correction adjusts $\mathcal{M}_{\alpha, \tau}^{(1)}$ with an additional factor penalizing remote locations, where the generators on average have to send their energy farther through the network than from a central bus.

$$
\mathcal{M}_{\alpha, \tau}^{(2)}=\left(\frac{\sum_{n \text { in region } \alpha}\left\langle P_{n, \tau}^{+}\right\rangle_{t} \bar{D}_{n}}{\sum_{m}\left\langle P_{m, \tau}^{+}\right\rangle_{t} \bar{D}_{m}}\right) \overline{\mathcal{K}}^{T}
$$

$\bar{D}_{n}$ is the average graph distance of the bus $n$, which is the mean distance to the other buses [34].

Flow tracing mean usage weights the attributions with a distribution determined from the average line loading of each generation type and region.

$$
\mathcal{M}_{\alpha, \tau}^{(3)}=\left(\frac{\sum_{l}\left\langle q_{l, \alpha, \tau} F_{l}\right\rangle_{t} \bar{L}_{l}}{\sum_{l^{\prime}}\left\langle F_{l^{\prime}}\right\rangle_{t} \bar{L}_{l^{\prime}}}\right) \overline{\mathcal{K}}^{T}
$$

It is similar to previously proposed pricing schemes based on flow tracing $[16,17]$.

Finally, Flow tracing usage measure distributes the capacity of each line by the usage measure from Eq. (22).

$$
\mathcal{M}_{\alpha, \tau}^{(4)}=\sum_{l} \overline{\mathcal{K}}_{l, \alpha, \tau}^{T}
$$

The four measures $\mathcal{M}_{\alpha, \tau}^{(1)}$ to $\mathcal{M}_{\alpha, \tau}^{(4)}$ for the 118-bus network model are illustrated in Figure 7.

The geometry factor which distinguishes $\mathcal{M}_{\alpha, \tau}^{(2)}$ from $\mathcal{M}_{\alpha, \tau}^{(1)}$ only has a marginal effect on the allocation, nevertheless it is still worth noting that the modification is mostly in direction of the results of more elaborate measures.

For most regions and generation types the simple measures $\mathcal{M}_{\alpha, \tau}^{(1 / 2)}$ agree quite well with the flow tracing based measures $\mathcal{M}_{\alpha, \tau}^{(3 / 4)}$. Incorporating the actual shares of the line loading by flow tracing turns out to have the largest effect for Region 14 which has a large consumption and exclusively conventional generation. Most of the power that is generated in Region 14 is consumed within few line kilometers so that actual network transmission is kept to a minimum, although the total power injected into the network is very high. This indicates that an average distance to the load centers instead of to all the buses in equal weights might be a better measure.

The capacity attributed to Region 14 is further reduced by taking the lineloading correlations into account, since its conventional generation is mainly 


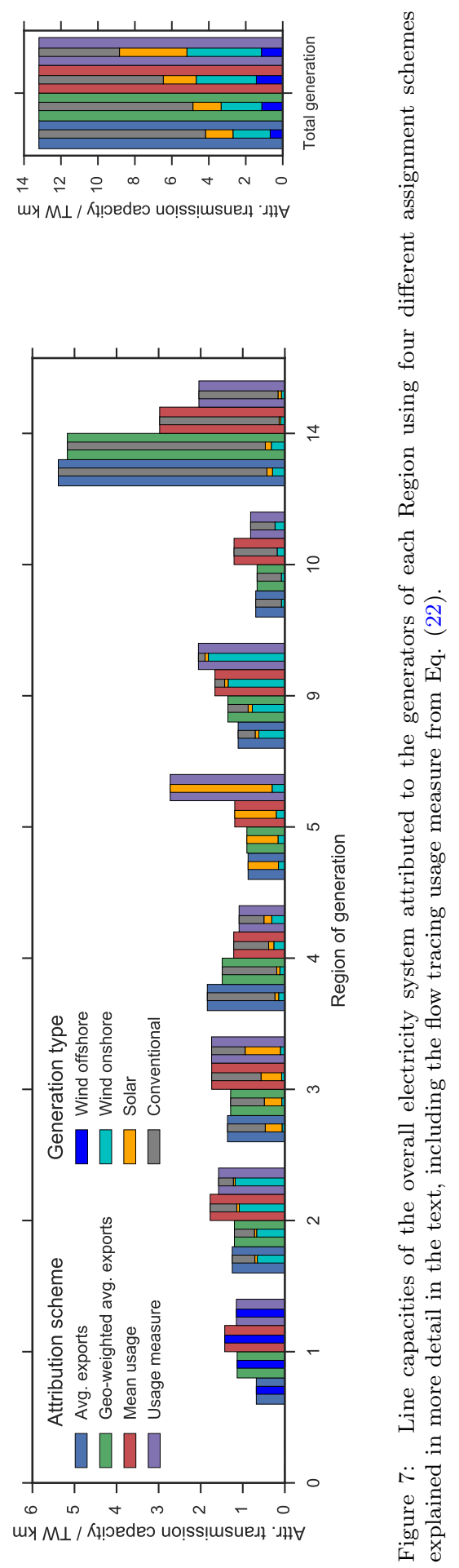


dispatched in times with low renewable generation and, thus, also small overall flows. But the most striking adjustment from $\mathcal{M}_{\alpha, \tau}^{(3)}$ to $\mathcal{M}_{\alpha, \tau}^{(4)}$ is that the capacity attributed to the solar generation in Region 5 doubles, which is due to the strong correlation between line-loading and solar flows already visible in the single line usage share of Fig. 5. The same effect can also be seen in the usage measure component for solar power generated in Region 3, only that the overall capacity for Region 4 balances out thanks to its conventionally dominated generation mix.

Overall, we find that wind generators are strongly affected by switching from a postage stamp pricing mechanism to a flow tracing based one, since the volume of wind energy in the network is often disproportionately high as a study by Brown et al., based on marginal participation, has already pointed out [9]. Additionally taking the correlation between line-loading and usage shares into account strongly impacts the capacities attributed to solar generation. The choice of a suitable capacity allocation measure thus depends on the range of system properties which should be represented. Whereas a simple postage stamp method might cover average imports and exports of the system participants, only more elaborate techniques based on flow allocation are able to incorporate the correlations and patterns emerging from the fluctuating imports and exports in large-scale electricity systems with a high share of renewable generation.

\section{Conclusion and Outlook}

Flow tracing is a well-known method to dissect the power flows on a network according to shares attributed to the network-injecting source nodes [14, 15$]$. Such an attribution of power flows and thus network usage has been proposed as an essential component for a fair allocation of both operational (for instance losses or stability measures) and grid infrastructure costs $[4,20,21,31]$. In the present contribution we show how a reformulation of the flow tracing method serves as powerful tool set to analyze the complex spatio-temporal patterns of generation, consumption and power flows in interconnected large-scale electricity systems, in particular those with a high share of renewable generation. At the point of injection into the network, the power flow can straightforwardly be assigned to a specific geographical location, mix of different generation technologies or any other attribution of interest. Following the composition of ingoing flow from the net generators through the network, the algorithm yields in an intuitive way the respective shares of the total power flow and of the outflow to the net consumers.

The potential of this method are illustrated in the context of the Scenario $2023 \mathrm{~B}$ of the 118-bus transmission benchmark case with renewable capacities [13]. We dissect the power flows into components associated with the geographical origin and generation technology (wind offshore/onshore, solar, conventional), yielding a selection of measures about the respective transmission system usage and the corresponding import-export relations between the system nodes. The need of incorporating relevant correlations in the aggregation 
procedure from full high-dimensional results to a lower-dimensional expression is discussed by comparing different transmission capacity usage measures assigned to the geographical regions of the benchmark network.

The discussion in the present paper suggests future work on different levels. From a technical point of view, it will be interesting to transfer the idea of more general in-partitions to alternative methods of flow allocation [7, 24, 35]. First steps in this direction have been taken by [9] for allocation methods based on power transfer distribution factors, but a rigorous discussion is still lacking in the literature. In the present paper we focus on illustrating the versatility of the reformulated flow tracing method by considering a well-defined and fully open benchmark system. We are confident that an approach as discussed here will be integrated into a state of the art tool set of system analysis and applied to scenarios of the current and future electricity system, helping to reveal patterns in the intricate interactions in this highly complex multi-level system. As shown for the test case, it is then challenging to select and adapt suitable measures from the the multitude of information which can be yielded using this technique.

\section{Acknowledgments}

We thank Tom Brown for constructive comments and discussions. The project underlying this report was supported by the German Federal Ministry of Education and Research under grant no. 03SF0472C. The responsibility for the contents lies with the authors. M.S. acknowledges support from Stiftung Polytechnische Gesellschaft Frankfurt and the Carlsberg Foundation.

\section{References}

\section{References}

[1] M. Aguado, R. Bourgeois, J. Bourmaud, J. Van Casteren, M. Ceratto, M. Jäkel, B. Malfliet, C. Mestdag, P. Noury, M. Pool, et al., Flow-based market coupling in the central western european region-on the eve of implementation, CIGRE, C5-204.

[2] European Network of Transmission System Operators for Electricity, ENTSO-E Ten Year Network Development Plan, Tech. rep., ENTSO-E, Brussels (2016).

[3] S. Hagspiel, C. Jägemann, D. Lindenberger, T. Brown, S. Cherevatskiy, E. Tröster, Cost-optimal power system extension under flow-based market coupling, Energy 66 (2014) 654-666. doi:10.1016/j.energy.2014.01. 025.

[4] CONSENTEC and Frontier Economics, Study on the further issues relating to the inter-TSO compensation mechanism (2006). 
[5] M. Neukirch, Protests against german electricity grid extension as a new social movement? a journey into the areas of conflict, Energy, Sustainability and Society 6 (1) (2016) 4. doi:10.1186/s13705-016-0069-9.

[6] A. J. Conejo, J. Contreras, D. A. Lima, A. Padilha-Feltrin, Zbus transmission network cost allocation, IEEE Transactions on Power Systems 22 (1) (2007) 342-349. doi:10.1109/TPWRS.2006.889138.

[7] Y. C. Chen, S. V. Dhople, Power divider, IEEE Transactions on Power Systems 31 (6) (2016) 5135-5143. doi:10.1109/TPWRS.2016.2519900.

[8] H. Rudnick, R. Palma, J. E. Fernandez, Marginal pricing and supplement cost allocation in transmission open access, IEEE Transactions on Power Systems 10 (2) (1995) 1125-1132. doi:10.1109/59.387960.

[9] T. Brown, Transmission network loading in Europe with high shares of renewables, Renewable Power Generation 9 (2015) 57-65.

[10] S. Jebaraj, S. Iniyan, A review of energy models, Renewable and Sustainable Energy Reviews 10 (4) (2006) 281 -311. doi:10.1016/j.rser. 2004. 09.004 .

[11] D. Connolly, H. Lund, B. Mathiesen, M. Leahy, A review of computer tools for analysing the integration of renewable energy into various energy systems, Applied Energy 87 (4) (2010) 1059 - 1082. doi:10.1016/j. apenergy.2009.09.026.

[12] J. Hörsch, T. Brown, S. Schramm, Spatial scale dependence in joint optimisation of generation and transmission for highly renewable scenarios, in: Proceedings of 15th Wind Integration Workshop, 2016.

[13] H. Barrios, A. Roehder, H. Natemeyer, A. Schnettler, A benchmark case for network expansion methods, in: PowerTech, 2015 IEEE Eindhoven, 2015, pp. 1-6. doi:10.1109/PTC.2015.7232601.

[14] J. Bialek, Tracing the flow of electricity, Generation, Transmission and Distribution, IEE Proceedings- 143 (4) (1996) 313-320. doi:10.1049/ ip-gtd:19960461.

[15] D. Kirschen, R. Allan, G. Strbac, Contributions of individual generators to loads and flows, Power Systems, IEEE Transactions on 12 (1) (1997) 52-60. doi:10.1109/59.574923.

[16] J. Bialek, Topological generation and load distribution factors for supplement charge allocation in transmission open access, Power Systems, IEEE Transactions on 12 (3) (1997) 1185-1193. doi:10.1109/59.630460.

[17] J. Bialek, Allocation of transmission supplementary charge to real and reactive loads, Power Systems, IEEE Transactions on 13 (3) (1998) 749-754. doi:10.1109/59.708576. 
[18] G. Strbac, D. Kirschen, S. Ahmed, Allocating transmission system usage on the basis of traceable contributions of generators and loads to flows, Power Systems, IEEE Transactions on 13 (2) (1998) 527-534. doi:10.1109/59. 667378.

[19] D. Kirschen, G. Strbac, Tracing active and reactive power between generators and loads using real and imaginary currents, Power Systems, IEEE Transactions on 14 (4) (1999) 1312-1319. doi:10.1109/59.801890.

[20] J. Bialek, S. Ziemianek, Tracing based transmission pricing of crossborder trades: fundamentals and circular flows, in: Power Tech Conference Proceedings, 2003 IEEE Bologna, Vol. 3, 2003, pp. 8 pp. Vol.3-. doi:10.1109/PTC.2003.1304456.

[21] J. Bialek, S. Ziemianek, R. Wallace, A methodology for allocating transmission losses due to cross-border trades, Power Systems, IEEE Transactions on 19 (3) (2004) 1255-1262. doi:10.1109/TPWRS.2004.831296.

[22] I. Pérez-Arriaga, L. Olmos Camacho, F. Rubio Odériz, Report on cost components of cross border exchanges of electricity (2002).

[23] L. O. Camacho, I. J. Perez-Arriaga, An assessment of inter-TSO compensation algorithms in the Internal Electricity Market of the European Union, Electrical Power \& Energy Systems 29 (2007) 699-712.

[24] D. A. Lima, A. Padilha-Feltrin, J. Contreras, An overview on network cost allocation methods, Electric Power Systems Research 79 (5) (2009) 750 758. doi:10.1016/j.epsr.2008.10.005.

[25] A. A. E. Ela, R. El-Sehiemy, Transmission usage cost allocation schemes, Electric Power Systems Research 79 (6) (2009) 926 - 936. doi:10.1016/ j.epsr.2008.12.005.

[26] F. J. Rubio-Oderiz, I. J. Perez-Arriaga, Marginal pricing of transmission services: a comparative analysis of network cost allocation methods, IEEE Transactions on Power Systems 15 (1) (2000) 448-454. doi:10.1109/59. 852158.

[27] J. Grainger, W. Stevenson, Power system analysis, McGraw-Hill series in electrical and computer engineering: Power and energy, McGraw-Hill, 1994.

[28] C. Vazquez, L. Olmos, I. J. Pérez-Arriaga, On the selection of the slack bus in mechanisms for transmission network cost allocation that are based on network utilization, in: Proceedings of the 15th Power Systems Computing Conference, PSCC, Seville, 2002.

[29] P. Kattuman, R. Green, J. Bialek, Allocating electricity transmission costs through tracing: a game-theoretic rationale, Operations Research Letters 32 (2) (2004) 114-120. doi:10.1016/S0167-6377(03)00095-6. 
[30] C. Achayuthakan, C. Dent, J. Bialek, W. Ongsakul, Electricity tracing in systems with and without circulating flows: Physical insights and mathematical proofs, Power Systems, IEEE Transactions on 25 (2) (2010) 10781087. doi:10.1109/TPWRS. 2009. 2037506.

[31] B. Tranberg, A. B. Thomsen, R. A. Rodriguez, G. B. Andresen, M. Schäfer, M. Greiner, Power flow tracing in a simplified highly renewable European electricity network, New Journal of Physics 17 (10) (2015) 105002.

[32] T. Brown, J. Hörsch, D. Schlachtberger, PyPSA: Python for Power System AnalysisarXiv:1707.09913v2.

[33] T. Soares, F. Pereira, H. Morais, Z. Vale, Cost allocation model for distribution networks considering high penetration of distributed energy resources, Electric Power Systems Research 124 (2015) 120 - 132. doi: 10.1016/j.epsr.2015.03.008.

[34] M. Newman, Networks: An Introduction, OUP Oxford, 2010.

[35] D. A. Lima, A. Padilha-Feltrin, Allocation of the costs of transmission losses, Electric Power Systems Research 72 (1) (2004) 13 - 20. doi:10. 1016/j.epsr. 2004.03.002. 